Background The preclinical evaluation of novel immune modulators for cancer treatment remains a challenge, as models require both, engraftment of human tumor cells and a compatible human immune cells. In previous experiments, we have demonstrated, that we can use either peripheral blood mononuclear cells (PBMC) or hematopoietic stem cells (HSC) to establish a humanized immune system with functional T-, B-, and NK cells, monocytes, and dendritic cells. However these models are limited by rarely matching HLA isotypes between tumor and immune cells. In this case study, we established a patient-derived xenograft (PDX) model from a patient with Head and Neck squamous cell cancer (HNSCC). After engraftment of HNSCC PDX, patients PBMC were used to humanize mice. By this procedure we successfully generated a patient-specific human tumor-immune cell model in mice with 100\% HLA-match. Model development included the comparison of PDX engraftment on mice with either HLA-matching or non HLA-matching PBMC's and purified $\mathrm{T}$ cells from different donors. Furthermore, these effects were investigated on humanized mice generated with HSC. Finally, we further validated the model by comparing treatment effects with the checkpoint inhibitor Nivolumab in the autologous immune cell PDX model with heterologous models.

Methods The HNSCC PDX was transplanted on NOG mice. After tumor engraftment mice were randomized in 6 groups, receiving PBMCs by i.v. transplantation either from the patient or from 5 well characterized donors (PDX patient PBMCs 100\% HLA matching, 5 donors with different HLA matching). In the last step, PDX were transplanted on humanized mice generated from 5 different HSC donors. Blood and tumor samples were analysed by FACS and IHC for immune cell infiltration and activation.

Results In the autologous huPBMC model, no interference with the proliferation of HNSCC PDX was seen. However, on mice humanized with donor PBMC's with a high HLA match, a strong stimulation of tumor proliferation compared to non-humanized mice was observed. On humanized mice, generated from 5 different HSC donors, HLA-matching seem to have a lower influence on engraftment. On mice humanized with PBMC from different donors, we observed a correlation of treatment effects with HLA match, with strong tumor growth inhibition in the mice with the best match. In the PDX tumors, infiltrating immune cells were detected by FACS and IHC analyses.

Conclusions We developed a humanized immune-PDX model enabling appropriate preclinical translational research on tumor immune biology and the evaluation of new therapies and combinations, as well as the identification and validation of biomarkers for immune therapy. Furthermore, results showed a correlation between immune therapy effects and HLA matching in preclinical models.

Disclosure Information M. Stecklum: A. Employment (full or part-time); Significant; EPO - Experimental Pharmacology \& Oncology Berlin-Buch GmbH. K. Klinghammer: None. A. Wulf-Goldenberg: A. Employment (full or part-time); Significant; EPO - Experimental Pharmacology \& Oncology Berlin-Buch GmbH. B. Brzezicha: A. Employment (full or parttime); Significant; EPO - Experimental Pharmacology \& Oncology Berlin-Buch GmbH. K. Jöhrens: None. J. Hoffmann: A. Employment (full or part-time); Significant; EPO - Experimental Pharmacology \& Oncology Berlin-Buch $\mathrm{GmbH}$.

\section{P03.15 SITE-SPECIFIC IMMUNE EVASION AND SUBSTANTIAL HETEROGENEITY WITHIN ENTITIES PROVIDE EVIDENCE FOR PERSONALIZED IMMUNOTHERAPY}

${ }^{1} \mathrm{M}$ Thelen* ${ }^{*}{ }^{1} \mathrm{~K}$ Wennhold, ${ }^{1} \mathrm{~J}$ Lehmann, ${ }^{1} \mathrm{E}$ Staib, ${ }^{1} \mathrm{MA}$ Garcia Marquez, ${ }^{2} \mathrm{P}$ Lohneis, ${ }^{3} \mathrm{~A}$ Lechner, ${ }^{2} \mathrm{~S}$ Wagener-Ryczek, ${ }^{2,4} \mathrm{PS}$ Plum, ${ }^{5} \mathrm{D}$ Pfister, ${ }^{6} \mathrm{~F}$ Dörr, ${ }^{7} \mathrm{D}$ Beutner, ${ }^{8} \mathrm{~F}$ Thangarajah, ${ }^{8} \mathrm{D}$ Ratiu, ${ }^{8} \mathrm{~W}$ Malter, ${ }^{2} \mathrm{~S}$ Merkelbach-Bruse, ${ }^{9} \mathrm{CJ}$ Bruns, ${ }^{2} \mathrm{~A}$ Quaas, ${ }^{10} \mathrm{MS}$ von Bergwelt-Baildon, ${ }^{9,11} \mathrm{HA}$ Schlößer. ${ }^{1}$ Center for Molecular Medicine Cologne, University of Cologne, Faculty of Medicine and University Hospital Cologne, Cologne, Germany; ${ }^{2}$ Institute of Pathology, University of Cologne, Faculty of Medicine and University Hospital Cologne, Cologne, Germany; ${ }^{3}$ Department of Otorhinolaryngology, Head and Neck Surgery, Grosshadern Medical Center, Ludwig Maximilians University, Munich, Munich, Germany; ${ }^{4}$ Department of General, Visceral, Cancer and Transplantation Surgery, University of Cologne, Faculty of Medicine and University Hospital Cologne, Cologne, Germany; ${ }^{5}$ Department of Urology, University of Cologne, Faculty of Medicine and University Hospital Cologne, Cologne, Germany; ${ }^{6}$ Department of Cardiothoracic Surgery, University of Cologne, Faculty of Medicine and University Hospital Cologne, Cologne, Germany; ' Department of Head and Neck Surgery, University of Göttingen, Göttingen, Germany; ${ }^{8}$ Department of Gynecology, University of Cologne, Faculty of Medicine and University Hospital Cologne, Cologne, Germany; ${ }^{9}$ Department of General, Visceral, Cancer and Transplantation Surgery, University of Cologne, Faculty of Medicine and University Hospital Cologne, Cologne, Germany; ${ }^{10}$ Department of Internal Medicine III, University Hospital, LMU Munich, Munich, Germany; ${ }^{11}$ Center for Molecular Medicine Cologne, University of Cologne, Faculty of Medicine and University Hospital Cologne, Cologne, Germany

\subsection{6/jitc-2020-ITOC7.54}

Background Immune-checkpoint inhibition (CKI) demonstrated remarkable therapeutic efficacy in several kinds of cancer. However, immune escape mechanisms lead to primary or secondary resistance in the majority of patients. Most predictive biomarkers failed, as the primary target of CKI is not the tumor cell itself, but the crosstalk between immune- and cancer cells. We aimed to characterize the immune evasion landscape in primary tumors across different entities.

Materials and Methods Expression of 32 immune-regulatory molecules on lymphocytes was analyzed in peripheral blood and tumor infiltrating lymphocytes (TILs) of 146 primary tumor patients across 10 different entities using flow cytometry. NanoString was applied to determine RNA expression of the respective ligands and 20 genes associated with antigen presentation. Expression of coinhibitory ligands on tumor cells was assessed by immunohistochemistry. To quantify the immune cell infiltration, digital pathology was used and the Immunoscore was generated for each patient.

Results While an increase of regulatory $\mathrm{T}$ cells was a common feature across all entities, we found site-specific differences regarding other lymphocyte subsets and expression of immune-regulatory molecules by TILs and tumor cells. Expression of co-inhibitory molecules on tumor infiltrating $\mathrm{T}$ cells accumulated especially in advanced stage cancers whereas immune cell infiltration was mainly associated with enhanced antigen presentation. Co-expression of multiple immune-inhibitory ligands was most frequent in colorectal, lung and ovarian carcinoma. Genes related to antigen presentation were frequently dysregulated in seminoma, liver and lung cancer.

Conclusions Immune evasion is a common feature of cancer and frequently detected co-occurrence of multiple mechanisms probably contributes to resistance against immunotherapy. We describe substantial heterogeneity regarding immune escape mechanisms between patients with the same primary tumor. Individualized immunotherapeutic strategies based on pretherapeutic evaluation of the immune evasion landscape might help to improve response to CKI.

Disclosure Information M. Thelen: None. K. Wennhold: None. J. Lehmann: None. E. Staib: None. M.A. Garcia Marquez: 
None. P. Lohneis: None. A. Lechner: None. S. Wagener-Ryczek: None. P.S. Plum: None. D. Pfister: None. F. Dörr: None. D. Beutner: None. F. Thangarajah: None. D. Ratiu: None. W. Malter: None. S. Merkelbach-Bruse: None. C.J. Bruns: None. A. Quaas: None. M.S. von Bergwelt-Baildon: B. Research Grant (principal investigator, collaborator or consultant and pending grants as well as grants already received); Significant; Astellas. F. Consultant/Advisory Board; Modest; Bristol-Myers Squibb. H.A. Schlößer: B. Research Grant (principal investigator, collaborator or consultant and pending grants as well as grants already received); Significant; Astra Zeneca.

\section{P03.16 FUNCTIONAL DEFECTS IN B-CELLS OF PATIENTS WITH VON-HIPPEL-LINDAU SYNDROME}

${ }^{1} \mathrm{~S}$ Theurich*, ${ }^{2} \mathrm{HJ}$ Becker, ${ }^{3} \mathrm{~K}$ Wennhold, ${ }^{3} \mathrm{H}$ Schlösser, ${ }^{3} \mathrm{~F}$ Marbach, ${ }^{3} \mathrm{M}$ Garcia-Marquez, ${ }^{3} \mathrm{~A}$ Shimabukuro-Vornhagen, ${ }^{3} \mathrm{~J}$ Schreml, ${ }^{1} \mathrm{M}$ von Bergwelt-Baildon. 'LMU Munich, Munich, Germany; ' University of Tokio, Tokio, Japan; ${ }^{3}$ University Hospital Cologne, Cologne, Germany

\subsection{6/jitc-2020-ITOC7.55}

Von-Hippel-Lindau (VHL)-disease is an inherited cancer syndrome characterized by a variety of benign and malignant tumors, which develop upon mutation of the second allele of the VHL-tumor suppressor gene. The VHL-protein (pVHL) regulates hypoxia-induced transcription factors (Hif) and by this plays a central role for metabolic cellular adaptations to hypoxic conditions. VHL/Hif regulation plays a well-established role in the development and function of immune cells and already VHL-haploinsufficiency can alter gene expression patterns. In contrast, little is known about primary immune cell functions in VHL-patients. In this study, we analyzed the functional capacity of CD40-stimulated B-cells to act as antigen-presenting cells. We confirmed mono-allelic VHL-gene mutations in B-cells from thirteen VHL-patients and found that their response to CD40-stimulation was significantly reduced. On a functional level this translated to an impaired ability to act as antigen presenting cells leading to impaired Tcell responses in vitro. Taken together, we demonstrate that VHL-haploinsufficiency deregulates B-cell functions following CD40-activation as a new aspect of VHL-syndrome. (The study was registered in the German Clinical Trial Registry (www.drks.de); ID: DRKS00012413).

Disclosure Information S. Theurich: B. Research Grant (principal investigator, collaborator or consultant and pending grants as well as grants already received); Modest; Verein VHL (von Hippel-Lindau) betroffener Familien e.V.. H.J. Becker: None. K. Wennhold: None. H. Schlösser: None. F. Marbach: None. M. Garcia-Marquez: None. A. Shimabukuro-Vornhagen: None. J. Schreml: None. M. von Bergwelt-Baildon: None.

\section{P03.17 UPA-PAI-1 HETEROMERS PROMOTE ADVANCED STAGES OF BREAST CANCER BY ATTRACTING PRO-TUMORIGENIC NEUTROPHILS}

${ }^{1} \mathrm{~B}$ Uhl ${ }^{*},{ }^{1} \mathrm{~L}$ Mittmann, ${ }^{1} \mathrm{~J}$ Dominik, ${ }^{1} \mathrm{~J}$ Schaubächer, ${ }^{1} \mathrm{C}$ Braun, ${ }^{1} \mathrm{R}$ Pick, ${ }^{1} \mathrm{M}$ Canis, ${ }^{2} \mathrm{~S}$ Kanse, ${ }^{3} \mathrm{~W}$ Weichert, ${ }^{1} \mathrm{M}$ Sperandio, ${ }^{1} \mathrm{~K}$ Lauber, ${ }^{1} \mathrm{~F}$ Krombach, ${ }^{1} \mathrm{CA}$ Reichel. ${ }^{1}$ University Hospital, Ludwig-Maximilians-University (LMU) Munich, München, Germany; ${ }^{2}$ University of Oslo, Oslo, Norway; ${ }^{3}$ Technical University of Munich, München, Germany

10.1136/jitc-2020-ITOC7.56
Background High tumor levels of urokinase-type plasminogen activator (uPA)-plasminogen activator inhibitor-1 (PAI-1) heteromers independently predict poor survival in early breast cancer. The pathogenetic role of this protein complex, however, remains largely obscure.

Materials and Methods Neutrophil trafficking was analyzed in orthotopic (multi-channel flow cytometry) and heterotopic (ear; multi-channel in vivo microscopy) mouse models of 4T1 breast cancer, in a mouse peritonitis assay (multi-channel flow cytometry), as well as in the mouse cremaster muscle (multi-channel in vivo microscopy). Cytokine expression in tumors was determined by multiplex ELISA. Phenotypic and functional properties of primary mouse neutrophils, microvascular endothelial cells (cell line bEnd.3), macrophages (cell line RAW 264.7), and breast cancer cells (cell line 4T1) were characterized in different in vitro assays. uPA/PAI-1 expression and neutrophil infiltration in human breast cancer samples were assessed by RNA sequencing, immunhistochemistry, and ELISA.

Results Here, we demonstrate that uPA-PAI-1 heteromerization multiplies the potential of the single proteins to attract protumorigenic neutrophils. To this end, tumor-released uPA-PAI1 utilizes very low density lipoprotein receptor and ERK mitogen-activated protein kinases to initiate a pro-inflammatory program in peritumoral macrophages. This promotes neutrophil trafficking to cancerous lesions and primes these immune cells towards a pro-tumorigenic phenotype, thus supporting tumor growth and metastasis. Blockade of uPA-PAI-1 heteromerization by a novel inhibitor effectively interfered with these events and prevented tumor progression.

Conclusions Our findings identify an already therapeutically targetable interplay between hemostasis and innate immunity that drives advanced stages of breast cancer. As a personalized immunotherapeutic strategy, blockade of uPA-PAI-1 heteromerization might be particularly beneficial for patients with highly aggressive uPA-PAI- $1^{\text {high }}$ tumors.

This study was supported by Deutsche Forschungsgemeinschaft (DFG), Sonderforschungsbereich (SFB) 914.

Disclosure Information B. Uhl: None. L. Mittmann: None. J. Dominik: None. J. Schaubächer: None. C. Braun: None. R. Pick: None. M. Canis: None. S. Kanse: None. W. Weichert: None. M. Sperandio: None. K. Lauber: None. F. Krombach: None. C.A. Reichel: None.

\section{P03.18 ABSTRACT WITHDRAWN}

\section{P03.19 EVALUATION OF IMMUNOGENICITY DIFFERENCES IN LLC1 AND GL261 TUMOR MODELS FOR EFFECTIVE CHEMO-IMMUNOTHERAPY TREATMENT}

K Zilionyte*, A Mlynska, V Pasukoniene. National Cancer Institute, Vilnius, Lithuania

\subsection{6/jitc-2020-ITOC7.57}

Background Tumor immunogenicity is a critical factor responsible for the limited success of cancer immunotherapy and determine the need for personalized treatment. Correct evaluation of effectiveness of cancer treatments and their combination is inseparable from the proper selection of the experimental tumor model. The lack of knowledge about the immunogenicity of animal tumor models makes it difficult to evaluate the efficacy of cancer immunotherapy and becomes 